\title{
An Application of the GHS Inequalities to Show the Absence of Phase Transition for Ising Spin Systems
}

\author{
C. J. Preston \\ Mathematical Institute, Oxford, U.K.
}

Received October 20, 1973

\begin{abstract}
We show that the GHS inequalities can be used instead of the Lee-Yang circle theorem to prove that there is no phase transition for the $v$-dimensional Ising model in the presence of a (non-zero) external field.
\end{abstract}

It has been shown by Ruelle [3] that there is no phase transition for the $v$-dimensional Ising model in the presence of a (non-zero) external field. A different proof of this result has been given by Lebowitz and Martin-Löf [2]. Both of these proofs use the Lee-Yang circle theorem and it is the object of this note to show that the result may be obtained by using the inequalities of Griffiths, Hurst and Sherman [1] rather than the circle theorem.

Let $\mathscr{C}$ denote the finite subsets of $\mathbb{Z}^{v}$ and let $\Phi: \mathscr{C} \rightarrow \mathbb{R}$ be a translation invariant, attractive pair potential, thus we have

$$
\begin{gathered}
\Phi(\emptyset)=0, \\
\Phi(A+x)=\Phi(A) \text { for all } A \in \mathscr{C}, x \in \mathbb{Z}^{v},
\end{gathered}
$$

$\Phi(A)=0 \quad$ if $\quad|A| \geqq 3, \quad$ (where $|A|$ denotes the cardinality of $A$ ), (3)

$$
\Phi(A) \geqq 0 \quad \text { if } \quad|A|=2 .
$$

We will also assume that $\Phi$ satisfies:

$$
\begin{gathered}
\sum_{0 \neq x \in \mathbb{Z}^{\nu}} \Phi(\{0, x\})<\infty, \\
\Phi(\{0\})+\sum_{0 \neq x \in \mathbb{Z}^{\nu}} \Phi(\{0, x\})=0 .
\end{gathered}
$$

Of course (6) just says that if we translate from "lattice gas" language to "spin" language then $\Phi$ corresponds to the Ising model in the absence of an external field. 
For $\lambda \in \mathbb{R}$ let $\Phi_{\lambda}$ denote the potential got by adding an external field of size $\lambda$ to $\Phi$, thus

$$
\Phi_{\lambda}(A)= \begin{cases}\Phi(A)+\lambda & \text { if }|A|=1 \\ \Phi(A) & \text { otherwise }\end{cases}
$$

Let $U_{\lambda}: \mathscr{C} \rightarrow \mathbb{R}$ be the energy corresponding to $\Phi_{\lambda}$, thus

$$
U_{\lambda}(A)=\sum_{B \subset A} \Phi_{\lambda}(B)=\sum_{B \subset A} \Phi(B)+\lambda|A| .
$$

For $\Lambda \in \mathscr{C}, \lambda \in \mathbb{R}$ let

$$
P_{\Lambda}(\lambda)=\frac{1}{|\Lambda|} \log \sum_{A \subset A} \exp U_{\lambda}(A) ;
$$

then if $\Lambda \uparrow \mathbb{Z}^{v}$ (in the sense of van Hove) we have $P_{\Lambda}(\lambda)$ converges (for all $\lambda \in \mathbb{R}$ ) to the pressure $P(\lambda)$. Using the FKG inequalities and the convexity of $P$ we have the following result of Lebowitz and Martin-Löf [2]:

Proposition 1. If $P$ is differentiable at $\lambda \in \mathbb{R}$ then phase transition does not occur for the interaction $\Phi_{\lambda}$.

(By the absence of phase transition we mean here that there exists only one infinite Gibbs state with potential $\Phi_{\lambda}$.)

By the Lee-Yang circle theorem it follows that $P$ is differentiable at $\lambda$ if $\lambda \neq 0$. We will now show that this also follows from the GHS inequalities.

Lemma 1. Let $I \subset \mathbb{R}$ be an open interval and for $n=1,2, \ldots$ let $f_{n}: I \rightarrow \mathbb{R}$ be convex and with $0 \leqq f_{n} \leqq 1$. Then there exists a subsequence $\left\{n_{j}\right\}$ such that $f_{n_{j}}(x)$ converges for all $x \in I$, and if we denote the limit by $f(x)$ then of course $f: I \rightarrow \mathbb{R}$ is also convex).

Proof. This is a well known result from real analysis.

Proposition 2. $P$ is differentiable at $\lambda$ if $\lambda \neq 0$.

Proof. Let $\tau:\{0,1\}^{\mathbb{Z}^{v}} \rightarrow\{0,1\}^{\mathbb{Z}^{v}}$ be the automorphism given by $\tau(A)=\mathbb{Z}^{v}-A$. Then $\tau$ induces an automorphism of the probability measures on $\{0,1\}^{\mathbb{Z}^{v}}$ and it is well-known (and easily checked) that this automorphism maps Gibbs states with potential $\Phi_{\lambda}$ into Gibbs states with potential $\Phi_{-\lambda}$. Thus we need only consider the case $\lambda<0$. For $\Lambda \in \mathscr{C}$ let $f_{\Lambda}=\frac{\partial P_{\Lambda}}{\partial \lambda} ;$ thus

$$
f_{\Lambda}(\lambda)=\frac{1}{|\Lambda|} \sum_{x \in \Lambda} \varrho_{\Lambda, \lambda}(\{x\}),
$$


where

$$
\varrho_{\Lambda, \lambda}(\{x\})=\frac{\sum_{x \in A \subset A} \exp U_{\lambda}(A)}{\sum_{A \subset A} \exp U_{\lambda}(A)} .
$$

Now if $\lambda<0$ then the inequalities of Griffiths, Hurst and Sherman [1] apply to $\Phi_{\lambda}$ and we thus get that $\varrho_{\Lambda, \lambda}(\{x\})$ is a convex function of $\lambda$ on $(-\infty, 0)$ and hence by Lemma 1 we can find $\Lambda_{n} \uparrow \mathbb{Z}^{v}$ and a convex function $f:(-\infty, 0) \rightarrow \mathbb{R}$ such that for all $\lambda<0$ we have both

$$
f_{\Lambda_{n}}(\lambda) \rightarrow f(\lambda) \text { and } P_{\Lambda_{n}}(\lambda) \rightarrow P(\lambda) \text { as } n \rightarrow \infty .
$$

Now let $\lambda<0$ and $\lambda_{0}<\lambda$; then

$$
P(\lambda)-P\left(\lambda_{0}\right)=\lim _{n \rightarrow \infty} \int_{\lambda_{0}}^{\lambda} f_{\Lambda_{n}}(t) d t=\int_{\lambda_{0}}^{\lambda} f(t) d t
$$

(where the last equality follows from the dominated convergence theorem, since $0 \leqq f_{A_{n}} \leqq 1$ ). But $f$ is convex and thus in particular continuous, hence by the fundamental theorem of calculus we have that $P$ is differentiable on $(-\infty, 0)$.

\section{References}

1. Griffiths, R. B., Hurst, C.A., Sherman, S.: J. Math. Phys. 11, 790-795 (1970)

2. Lebowitz, J.L., Martin-Löf, A.: Commun. math. Phys. 25, 276-282 (1972)

3. Ruelle, D.: Ann. Phys. 69, 364-374 (1972)

Communicated by G. Gallavotti

C. J. Preston Mathematical Institute 24-29 St. Giles Oxford, U.K. 
